\title{
Dynamic nuclear polarization in samarium-doped lanthanum magnesium nitrate*
}

\author{
Charles E. Byvik \\ NASA-Langley Research Center, Hampton, Virginia 23665 \\ David S. Wollan \\ Virginia Polytechnic Institute and State University, Blacksburg, Virginia 24061
}

(Received 31 October 1973)

\begin{abstract}
We report theoretical and experimental investigations of the dynamic nuclear polarization (DNP) and nuclear spin-lattice relaxation of protons in single crystals of lanthanum magnesium nitrate (LMN) weakly doped $(\approx 0.1-1.1$-mole $\%)$ with samarium ions $(\mathrm{LMN}: \mathrm{Sm})$ at $9.1 \mathrm{GHz}$ and liquid-helium temperatures. Theoretical expressions for DNP and nuclear relaxation are given for a homogeneously broadened ESR line in the high-temperature limit. DNP effects due to the electron dipole-dipole reservoir (EDDR) are predicted for the case of well-resolved satellites. Our DNP experiments on LMN:Sm are in general agreement with the conventional theory, but no evidence for direct EDDR effects on DNP with well-resolved satellites is seen, despite suggestive nuclear-relaxation data. Various explanations for this disagreement are proposed. We suggest that these EDDR effects may be seen in crystals of $\approx 3-5$-mole $\% \mathrm{LMN}: \mathrm{Sm}$ or in equivalent systems.
\end{abstract}

\section{INTRODUCTION}

We report theoretical and experimental investigations of the dynamic nuclear polarization $(\mathrm{DNP})^{1-11}$ and nuclear spin-lattice relaxation $^{1-4,7,9,11-16}$ of protons in single crystals of samarium-doped lanthanum magnesium nitrate (LMN: Sm) at $9.1 \mathrm{GHz}$ and liquid-helium temperatures. This material is of interest because the satellite electron spin resonance (ESR) transitions are well resolved from the main ESR line at $X-$ band microwave frequencies. The limit of wellresolved satellites often occurs in other materials at higher frequencies $\approx 50-70 \mathrm{GHz}$, where the ESR microwave techniques are more difficult. ${ }^{1,10}$ Larson and Jeffries ${ }^{17}$ have studied the electron spinlattice relaxation of Sm in LMN. They found no evidence of a phonon bottleneck, which is a complicating factor in the analysis of DNP experiments. ${ }^{2,3,5,6}$ Thus LMN : Sm appears useful for experimental tests of DNP theories in the limit of well-resolved satellites. One possible disadvantage is the high rejection ratio of the $\mathrm{Sm}$ ions in aqueous growing solutions of LMN: Sm, which may limit the maximum attainable Sm doping percent, and could indicate non random Sm spatial distributions in the LMN host lattice. We are particularly interested in the possibility of observing DNP effects due to the electron dipole-dipole reservoir (EDDR). 3, 4, 11, 18, 19

In Sec. II we give theoretical expressions for DNP and nuclear relaxation for a homogeneously broadened ESR line in the high-temperature limit and in the absence of a phonon bottleneck, incorporating rapid nuclear spin diffusion. These results, more complete than many previous versions, allow us to predict a new DNP effect for the case of well-resolved satellites. Our experimental work in $0.1-1.1-$ mole $\% \mathrm{LMN}: \mathrm{Sm}$, presented and discussed in Secs. III-VI, shows no evidence for direct EDDR DNP effects. We propose reasons for this, and suggest that they may be seen in $\approx 3-5$-mole \% LMN:Sm crystals, or in equivalent systems.

\section{THEORY}

A. Spin-temperature theory

We consider a system of $N_{n}$ spin- $-\frac{1}{2}$ nuclei per $\mathrm{cm}^{3}$ in an insulating, diamagnetic solid which is weakly doped with $N_{e}$ paramagnetic impurities (electrons, hereafter) per $\mathrm{cm}^{3}$ with effective spin $\frac{1}{2}$. For example, in our experiments the host matrix LMN $\left(\mathrm{La}_{2} \mathrm{Mg}_{3}\left(\mathrm{NO}_{3}\right)_{12} \cdot 24 \mathrm{H}_{2} \mathrm{O}\right)$ has 48 hydrogen nuclei (protons, hereafter) per molecule, and the electrons are the Sn impurity ions. We assume that all nuclei other than the protons have zero spin or negligible effect (except as noted)。 It is well known that saturation of the main and satellite ESR transitions polarizes the nuclei..$^{1-11}$ A correct treatment of magnetic resonance saturation in solids ${ }^{11,18,19}$ requires the use of the rotating reference frame and the magnetic dipole-dipole reservoir of the spins. These ideas have been extended to DNP. ${ }^{3,4,8,11,20}$ We summarize the results here, as the discussion of assumptions and tedious calculations have been given elsewhere. ${ }^{4,11,20}$

Let the electron (nuclear) spins, each of gyromagnetic ratio $\gamma_{e}\left(\gamma_{n}\right)$, be subject to a uniform and constant magnetic field $\overrightarrow{\mathrm{H}}_{0}$ in the $z$ direction, and to a small ESR rf field of amplitude $2 H_{1}$ oscillating in the $x$ direction at angular frequency $\omega \approx \omega_{e}$, where $\omega_{e}\left(=-\gamma_{e} H_{0}\right)$ is the electron Larmor frequency. We assume $\gamma_{e}$ to be isotropic. The effect of a nonsaturating NMR rf field near the nuclear Larmor frequency $\omega_{n}\left(=-\gamma_{n} H_{0}\right)$, used to measure the 
DNP, may be neglected here. ${ }^{21}$ Usually one finds $\omega_{e} \approx\left(10^{2}-10^{3}\right) \omega_{n} \gg \omega_{n}$. If $S_{z}\left(=\sum_{i=1}^{N_{e}} S_{z}^{i}\right)$ and $I_{z}$ $\left(=\sum_{f=1}^{N_{n}} I_{z}^{j}\right)$ are the $z$ components of the total electron and nuclear-spin operators, then the Hamiltonian $\mathcal{H}$ for this system may be witten ${ }^{4,11,20}$

$$
\begin{aligned}
\mathscr{H}= & \hbar \omega_{e} S_{z}+\hbar \omega_{n} I_{z}+\mathcal{H}_{S S}+\mathcal{H}_{S I}+\mathcal{H}_{I I} \\
& +2 \hbar \omega_{1} S_{x} \cos \omega t+\mathcal{H}_{S L} .
\end{aligned}
$$

The first two terms in Eq. (1) are the electron and nuclear Zeeman energies; $\mathcal{H}_{S S}, \mathcal{H}_{S I}$, and $\mathcal{H}_{I I}$ are the magnetic dipole-dipole interactions ${ }^{11,20,22}$ between the electrons, between the electrons and nuclei, and between the nuclei, respectively; the sixth term is the electronic interaction with the ESR rf field, where $\omega_{1}=-\gamma_{e} H_{1}$; and $\mathcal{H}_{S L}$ is the interaction of the spins with the lattice phonons. It is assumed that the homogeneous broadening of the ESR line completely dominates the inhomogeneous broadening. ${ }^{23}$

We transform to the rotating frame with the unitary operator $R(t)=e^{i \omega S_{z} t}$, where $t$ is time. The basic assumption ${ }^{11,19,20}$ of the spin-temperature theory is that the system may be described by a quasicanonical, time-dependent density matrix $\rho^{R}(t)$ in the rotating frame:

$$
\begin{aligned}
\rho^{R}(t) \propto & \exp \left[-\alpha(t) \hbar \Delta S_{z}-\beta(t) \hbar \omega_{n} I_{z}\right. \\
& \left.-\gamma(t) \mathcal{H}_{S S}^{0}\right],
\end{aligned}
$$

where $\alpha(t), \beta(t)$, and $\gamma(t)$ are time-dependent, inverse spin temperatures of the electron Zeeman reservoir (EZR), nuclear Zeeman reservoir (NZR), and electron dipole-dipole reservoir (EDDR). We use the frequency difference $\Delta=\omega_{e}-\omega$, and note that $\mathcal{H}_{S S}^{0}$ is that part of $\mathcal{H}_{S S}$ which commutes with $S_{z}$. The terms in the transformed Hamiltonian $R(t) \mathcal{H C} R(t)^{-1}$ not used in Eq. (2) a re small perturbations which cause transitions between the three main reservoirs and with the lattice; the $\mathcal{H}_{I I}$ term is responsible for spin diffusion (see below). The transition rates have been calculated in the hightemperature limit, in which Eq. (2) is expanded to lowest order. This approximation makes the calculation tractable, and corresponds to the experimental conditions encountered in this work. The results are ${ }^{20}$

$$
\begin{aligned}
\frac{d \alpha(t)}{d t}= & -W^{0}(\Delta)(\alpha-\gamma)+\frac{N_{n}}{N_{e}} \frac{1}{\Delta} W^{-}\left(\omega_{n}-\Delta\right)\left[\omega_{n} \beta-\Delta \alpha-\left(\omega_{n}-\Delta\right) \gamma\right]-\frac{N_{n}}{N_{e}} \frac{1}{\Delta} W^{+}\left(\omega_{n}+\Delta\right)\left[\omega_{n} \beta+\Delta \alpha-\left(\omega_{n}+\Delta\right) \gamma\right] \\
& -\frac{1}{T_{e}}\left(\alpha-\frac{\omega_{e}}{\Delta} \beta_{L}\right) \\
\frac{d \beta(t)}{d t}= & -W^{d}(\beta-\gamma)-\frac{1}{\omega_{n}} W^{-}\left(\omega_{n}-\Delta\right)\left[\omega_{n} \beta-\Delta \alpha-\left(\omega_{n}-\Delta\right) \gamma\right]-\frac{1}{\omega_{n}} W^{+}\left(\omega_{n}+\Delta\right)\left[\omega_{n} \beta+\Delta \alpha-\left(\omega_{n}+\Delta\right) \gamma\right] \\
& -\frac{1}{T_{n}}\left(\beta-\beta_{L}\right) \\
\frac{d \gamma(t)}{d t}= & \frac{\Delta^{2}}{\omega_{L}^{2}} W^{0}(\Delta)(\alpha-\gamma)+\frac{N_{n}}{N_{e}} \frac{\omega_{n}^{2}}{\omega_{L}^{2}} W^{d}(\beta-\gamma)+\frac{N_{n}}{N_{e}} \frac{\left(\omega_{n}-\Delta\right)}{\omega_{L}^{2}} W^{-}\left(\omega_{n}-\Delta\right)\left[\omega_{n} \beta-\Delta \alpha-\left(\omega_{n}-\Delta\right) \gamma\right] \\
& +\frac{N_{n}}{N_{e}} \frac{\left(\omega_{n}+\Delta\right)}{\omega_{L}^{2}} W^{+}\left(\omega_{n}+\Delta\right)\left[\omega_{n} \beta+\Delta \alpha-\left(\omega_{n}+\Delta\right) \gamma\right]-\frac{1}{T_{d}}\left(\gamma-\beta_{L}\right)
\end{aligned}
$$

where $\beta_{L}$ is the inverse temperature of the lattice (assumed equal to that of the bath), and $\omega_{L}^{2}$ $=\left[\operatorname{Tr}\left(\mathcal{H C}_{S S}^{0}\right)^{2} / \hbar^{2} \operatorname{Tr}\left(S_{z}\right)^{2}\right]$ is $\frac{1}{3}$ of the second moment of the ESR line. ${ }^{4,11} T_{e}, T_{n}$, and $T_{d}$ are the spinlattice relaxation times of EZR, NZR, and EDDR, respectively. Abragam and Borghini ${ }^{4}$ take $\left(T_{e} / T_{d}\right)$ $\approx 2-3$, which we assume henceforth (except as noted). It is assumed that there is no phonon bottleneck between EZR or EDDR and the bath. The transition rate $W^{0}(\Delta)$ of the main ESR line is

$$
W^{0}(\Delta)=\pi \omega_{1}^{2} g(\Delta),
$$

where the line-shape function $g(\Delta),{ }^{11,20,22}$ normal- ized to unity, peaks at $\Delta=0$. The two weaker satellite transition rates

$$
W^{ \pm}\left(\omega_{n} \pm \Delta\right)=\pi\left(\epsilon^{2} / \omega_{n}^{2}\right) \omega_{1}^{2} g\left(\omega_{n} \pm \Delta\right)
$$

peak at $\omega_{n} \pm \Delta=0$, and correspond to simultaneous electron and nuclear transitions. The rf independent term ${ }^{24}$

$$
W^{d}=\pi \epsilon^{2} g\left(\omega_{n}\right)
$$

gives the rate of energy exchange between NZR and EDDR. 7, 8, 13-15

We assume that the random distribution of electrons in the host lattice may be approximated by a 
uniform electron distribution. Thus we may divide the sample into average shells-of-influence of outer radius $R=\left(3 / 4 \pi N_{e}\right)^{1 / 3}$. The inner radius is the diffusion barrier radius $d$. For small electronimpurity concentrations, a given electron affects directly only the nuclei within its own shell. Since we are treating the electron system as homogeneous, each electron may be described by the two temperatures $\alpha(t)$ and $\gamma(t)$ which characterize the electronic system as a whole. We then calculate the sample average for $\beta(t)$ in Eqs. (3)-(6) by averaging over one shell-of-influence. ${ }^{20}$ The factor $\epsilon^{2}$, which is the shell-of-influence angular and radial average value of the relevant perturbation term from $\mathfrak{H}_{S I}$, is found to be

$$
\epsilon^{2}=\frac{3\left(\gamma_{n} \gamma_{e} \hbar\right)^{2}}{10 d^{3} R^{3}}
$$

This calculation, which ignores the crystal structure, is valid in the rapid diffusion limit of spin diffusion which is applicable to the conditions of our experiments, and has been justified elsewhere. ${ }^{1-4,9,11-16}$ The diffusion barrier radius $d$ in Eq. (7) has been estimated by Blumberg ${ }^{12}$ and Khutsishvili ${ }^{9}$ to be $d \approx\left(\gamma_{e} / \gamma_{n}\right)^{1 / 3} a \approx 10 \AA$, where $a$ is the average internuclear distance. Jeffries $e t$ $a l .^{1-3}$ take $d$ as the distance from the electron to nearest proton (4.36 $\AA$ in LMN). Recent theoreti$\mathrm{cal}^{25}$ and experimental ${ }^{26}$ work supports the latter choice, which we make henceforth.

\section{B. Nuclear spin-lattice relaxation}

The nuclei relax to the lattice directly by the $\left(T_{n}\right)^{-1}$ term in Eq. (3b), and indirectly through EDDR by terms involving $W^{d}$. The term $\left(T_{n}\right)^{-1}$ can be written

$$
\frac{1}{T_{n}}=\frac{1}{T_{n}^{Z}}+\frac{1}{T_{n}^{L}}
$$

where $\left(T_{n}^{Z}\right)^{-1}$ is relaxation through EZR by the desired impurity (e.g., Sm in our case). The nuclear leakage relaxation rate $\left(T_{n}^{L}\right)^{-1}$ is caused by undesired paramagnetic impuricies and other modes of NZR relaxation. In the rapid-diffusion limit, one find $\mathrm{s}^{1-4,9,11-16}$

$$
\frac{1}{T_{n}^{Z}}=\frac{\epsilon^{2} T_{e}}{1+\left(\omega_{n} T_{e}\right)^{2}}
$$

and a similar expression for $W^{d}$ with the electron spin-spin relaxation time ${ }^{22} T_{2 e}$ replacing $T_{e}$, if one assumes a Lorentzian $g\left(\omega_{n}\right)$ in Eq. (6). ${ }^{24}$ When . $\left(\omega_{n} T_{e}\right)^{2},\left(\omega_{n} T_{2 e}\right)^{2} \gg 1$, these become ${ }^{27}$

$$
\begin{aligned}
& \frac{1}{T_{n}^{Z}}=\frac{3}{10}\left(\frac{g_{e} \mu_{B}}{H_{0}}\right)^{2} \frac{1}{d^{3} R^{3}} \frac{1}{T_{e}}, \\
& W^{d}=\frac{3}{10}\left(\frac{g_{e} \mu_{B}}{H_{0}}\right)^{2} \frac{1}{d^{3} R^{3}} \frac{1}{T_{2 e}},
\end{aligned}
$$

where $g_{e}$ is the electron $g$ factor, $\mu_{B}$ is the Bohr magneton, and we use $g_{e} \mu_{B}=\left|\gamma_{e}\right| \hbar$. We define $S^{d}$ $\equiv W^{d} T_{n}$. If $\left(T_{n}^{Z} / T_{n}^{L}\right) \ll 1$, then Eqs. (10) and (11) lead to

$$
S^{d}=W^{d} T_{n}=\left(T_{e} / T_{2 e}\right) .
$$

During a nuclear-relaxation experiment, the time evolution of the NZR temperature $\beta(t)$ is described by Eqs. (3b) and (3c) with $W^{0}, W^{ \pm}$all zero. The solutions to these equations contain two time constants. In the limit $T_{n} \gg T_{d},\left(N_{n} \omega_{n}^{2} / N_{e} \omega_{L}^{2}\right) \gg 1$, the measured nuclear spin-lattice relaxation time $T_{n}^{m}$ will always be the longer of the two time constants, given by

$$
\frac{1}{T_{n}^{m}}=\frac{1}{T_{n}}+\frac{W^{d}}{1+\left(N_{n} \omega_{n}^{2} / N_{e} \omega_{L}^{2}\right) W^{d} T_{d}} .
$$

This result is easily obtained, since EDDR comes to quasiequilibrium $[(d \gamma / d t) \approx 0]$ in a time $t \ll T_{n}^{m}$. We can solve Eq. $(3 \mathrm{c})$ for $\gamma(t)$ and substitute into Eq. (3b) to deduce

$$
\frac{d \beta(t)}{d t}=-\frac{1}{T_{n}^{m}}\left[\beta(t)-\beta_{L}\right],
$$

with $T_{n}^{m}$ given by Eq. (13). These results agree with Khutsishvili ${ }^{14}$ and Van den Heuvel et al. ${ }^{15}$ in the appropriate limit. If the $W^{d}$ terms predominate, so that $T_{n}^{m} \ll T_{n}$, one cannot measure $T_{n}$ in a nuclear-relaxation experiment.

\section{Dynamic nuclear polarization at the satellite peaks}

We now consider the limit of well-resolved satellites when $W^{ \pm} \neq 0, W^{0}=0=W^{\mp}$, and calculate the DNP enhancement $E \equiv\left(\beta / \beta_{L}\right)$ at the satellite peaks $\left(\omega_{n} \pm \Delta=0\right)$, where Eqs. (3a) $-(3 \mathrm{c})$ are considerably simplified. For the limit $T_{n} \gg T_{e} \approx T_{d},\left(N_{n} \omega_{n}^{2} /\right.$ $\left.N_{e} \omega_{L}^{2}\right) \gg 1$, EZR and EDDR come to quasiequilibrium $[(d \alpha / d t) \approx 0 \approx(d \gamma / d t)]$ in a time $t \ll T_{n}^{m}$, so we may solve Eqs. (3a) and (3c) for $\alpha(t)$ and $\gamma(t)$, and substitute into $\mathrm{Eq}$. (3b) to get

$$
\frac{d E(t)}{d t}=-\frac{1}{\tau_{\mathrm{DNP}}}\left[E(t)-E_{\mathrm{ss}}\right],
$$

where the sample average, steady-state enhancement $E_{s s}$ is

$$
E_{\text {ss }}=\frac{1+S_{m}^{ \pm}\left[\mp\left(\omega_{e} / \omega_{n}\right)+f_{e}^{m}\right]}{1+S_{m}^{ \pm}\left(1+f_{e}^{m}\right)},
$$

and the DNP pump time $\tau_{\mathrm{DNP}}$ is given by

$$
\frac{1}{\tau_{\mathrm{DNP}}}=\frac{1}{T_{n}^{m}}\left(\frac{1+S_{m}^{ \pm}\left(1+f_{e}^{m}\right)}{1+S_{m}^{ \pm} f_{e}^{m}}\right) \text {. }
$$

We use the DNP leakage factor $f_{e}^{m} \equiv\left(N_{n} T_{e} / N_{e} T_{n}^{m}\right)$ and the satellite saturation parameter $S_{m}^{ \pm} \equiv W^{ \pm} T_{n}^{m}$ 。 At the satellite peaks $E_{\mathrm{ss}}$ approaches $\left(\mp \omega_{e} / \omega_{n}\right) /$ $\left(1+f_{e}^{m}\right)$ at strong saturation $\left(S_{m}^{ \pm} \gg 1\right)$, attaining the ideal enhancement ${ }^{28} E_{i}=\left(\mp \omega_{e} / \omega_{n}\right)$ if there is negligible DNP leakage $\left(f_{e}^{m} \ll 1\right)$. Our results are simi- 
lar to those of Jeffries et al. ${ }^{1-3}$ and Borghini, ${ }^{5}$ but include the important $W^{d}$ contributions to $T_{n}^{m}$ which they did not consider. However, their results are not restricted to the high-temperature limit, and include the effect of a phonon bottleneck. Our calculation can be extended to include the latter, but calculation of EDDR properties at very low temperatures is an unsolved problem. Neither is relevant for our experiments.

Several relations involving the DNP leakage factors are now deduced. We define $f_{e} \equiv\left(N_{n} T_{e} /\right.$ $\left.N_{e} T_{n}\right), f_{d} \equiv\left(T_{d} / T_{e}\right) f_{e}, f_{e}^{m}=\left(T_{n} / T_{n}^{m}\right) f_{e}, C_{n} \equiv \omega_{n}^{2} / \omega_{L}^{2}$, and recall $S^{d} \equiv W^{d} T_{n}$. Equation (13) can then be rewritten as

$$
T_{n}^{m}=T_{n} \frac{1+S^{d} f_{d} C_{n}}{1+S^{d}\left(1+f_{d} C_{n}\right)} .
$$

If $S^{d} \lesssim 1$, or if $f_{d} C_{n} \gg 1$, one gets $T_{n}^{m} \approx T_{n}$, and $f_{e}^{m}$ $\approx f_{e}$, so that EDDR does not significantly affect the nuclear relaxation or the DNP leakage factors. We now consider the two possibilities for the case of well-resolved satellites $\left(C_{n} \gg 1\right)$ when $S^{d} \gg 1$, $f_{d} C_{n} \leqslant 1$. First, if $S^{d} f_{d} C_{n} \gg 1$, one finds $\left(T_{n}^{m} / T_{n}\right)$ $\approx\left(f_{d} C_{n}\right)$ and $f_{e}^{m} \approx\left(T_{e} / T_{d} C_{n}\right) \ll 1$. Second, if $S^{d} f_{d} C_{n}$ $\leqslant 1$, we get $\left(T_{n}^{m} / T_{n}\right) \approx\left(S^{d}\right)^{-1}$ and $f_{e}^{m} \approx f_{e} S^{d} \ll 1$. Hence, for well-resolved satellites, either $f_{e}^{m} \approx f_{e}$ (if $S^{d}$ ミ) or $f_{e}^{m} \ll 1$ (if $S^{d} \gg 1$ ).

We can use Eqs. (8) and (10) to show

$$
f_{e}=\frac{2}{5} \pi \frac{N_{n}}{d^{3}}\left(\frac{g_{e} \mu_{B}}{H_{0}}\right)^{2}\left(1+\frac{T_{n}^{Z}}{T_{n}^{L}}\right) .
$$

If $\left(T_{n}^{Z} / T_{n}^{L}\right) \ll 1$, this can be written

$$
f_{e}=\frac{K}{d^{3}}\left(\frac{g_{\theta}}{H_{0}}\right)^{2},
$$

where $K=8.56 \times 10^{-18}$ (cgs units) for LMN. Thus $f_{e}$ is independent of $N_{e}$ when nuclear leakage relaxation is negligible. For the conditions of our experiments in LMN : Sm $\left(g_{e}=0.73, H_{0}=8900 \mathrm{Oe}, d=4.36\right.$ $\AA$ ), we get $f_{e} \approx 0.007 \ll 1$. In view of the last paragraph, this means that we should observe $f_{c}^{m} \ll 1$ in LMN : Sm, assuming $\left(T_{n}^{L}\right)^{-1}$ to be negligible. Using the same approximations and $\left(T_{e} / T_{d}\right) \approx 2$, we find for $\mathrm{LMN}: \mathrm{Sm}$ that

$$
f_{d} C_{n} \approx 0.5 / H_{L}^{2},
$$

where $H_{L}^{2}=\omega_{L}^{2} / \gamma_{e}^{2}$. In our experiments we have $H_{L}^{2} \approx 1(\mathrm{Oe})^{2}$, so that $f_{d} C_{n} \approx 0.5$.

D. EDDR effects on dynamic nuclear polarization

At the satellite peaks, EDDR affects the DNP indirectly through $T_{n}^{m}$ and the leakage factor $f_{e}^{m}$. However, away from the peaks $\left(\omega_{n} \pm \Delta \neq 0\right)$, EDDR may contribute explicitly to the DNP. As in Sec. II C, we assume that EZR and EDDR come to quasiequilibrium in a time $t \ll T_{n}^{m}$, so we may solve Eqs. (3a) and (3c) for $\alpha(t)$ and $\gamma(t)$, and substitute into Eq. (3b), retaining all terms. After a tedious calculation, we get Eq. (15) with the $E_{\text {ss }}$ and $\tau_{\mathrm{DNP}}$ expressions

$$
E_{\mathrm{ss}}=A / B \text {, }
$$

where

$$
\begin{aligned}
A= & 1+S^{d}\left(1+f_{d} C_{n}\right)+S^{0}\left(1+C_{\Delta} R\right)+S^{+}\left[-\left(\omega_{e} / \omega_{n}\right)+\left(\omega_{n}+\Delta\right) / \omega_{n}+f_{e}+f_{d} C_{+}\right]+S^{-}\left[+\left(\omega_{e} / \omega_{n}\right)+\left(\omega_{n}-\Delta\right) / \omega_{n}\right. \\
& \left.+f_{e}+f_{d} C_{-}\right]+\left[S^{0}\left(S^{d}+S^{+}+S^{-}\right)+f_{e}\left(S^{+}+S^{-}\right) S^{d}\right]\left[\left(\omega_{e} / \omega_{n}\right)\left(\omega_{n} \Delta / \omega_{L}^{2}\right) R+1+f_{d} C_{n}\right]
\end{aligned}
$$

and

$$
\begin{aligned}
B= & 1+S^{d}\left(1+f_{d} C_{n}\right)+S^{0}\left(1+C_{\Delta} R\right)+S^{+}\left(1+f_{e}+f_{d} C_{+}\right)+S^{-}\left(1+f_{e}+f_{d} C_{-}\right)+\left[S^{0}\left(S^{d}+S^{+}+S^{-}\right)\right. \\
& \left.+f_{e}\left(S^{+}+S^{-}\right) S^{d}\right]\left[C_{\Delta} R+1+f_{d} C_{n}\right] ;
\end{aligned}
$$

and

$$
\frac{1}{\tau_{\mathrm{DNP}}}=\frac{1}{T_{n}} \frac{B}{C}
$$

where

$$
C=1+S^{d}\left(f_{d} C_{n}\right)+S^{0}\left(1+C_{\Delta} R\right)+S^{+}\left(f_{e}+f_{d} C_{+}\right)+S^{-}\left(f_{\theta}+f_{d} C_{-}\right)+\left[S^{0}\left(S^{d}+S^{+}+S^{-}\right)+f_{e}\left(S^{+}+S^{-}\right) S^{d}\right]\left(f_{d} C_{n}\right) .
$$

We use the saturation parameters $S^{d} \equiv W^{d} T_{n}, S^{0}$ $\equiv W^{0} T_{e}, S^{ \pm} \equiv W^{ \pm} T_{n}$, the relative heat capacity ratios $C_{n} \equiv \omega_{n}^{2} / \omega_{L}^{2}, C_{\Delta} \equiv \Delta^{2} / \omega_{L}^{2}, C_{ \pm} \equiv\left(\omega_{n} \pm \Delta\right)^{2} / \omega_{L}^{2}$, and the electron relaxation ratio $R \equiv T_{d} / T_{e}$. Negligible $S^{ \pm} S^{\mp}$ terms are omitted in Eqs. (21b), (21c), and (22b).

We now specialize Eqs. (21a)--(21c) to the case of well-resolved satellites, where no more than one of the three parameters $S^{0}, S^{ \pm}$may be nonzero for a given value of $\Delta$. In this case Eqs. (21a)-(21c) reduce to $\mathrm{Eq}$. (16) if one is at the satellite peaks $\left(\omega_{n} \pm \Delta=0\right)$, or if $S^{d} \ll 1$.

Let us now consider possible direct EDDR effects on DNP away from the satellite peaks in the limit $S^{d} \gg 1$. First, we examine the case of strong saturation of the main ESR line $\left(S^{0} \gg 1\right)$, assuming 
TABLE I. Summary of experimental and theoretical parameters. The crystal designation is listed first: item 1 is the temperature; item 2 is the ESR spectrometer microwave frequency; item 3 is the angle between the applied magnetic field $\overrightarrow{\mathrm{H}}_{0}$ and the crystal symmetry axis; item 4 is the measured proton spin-lattice relaxation time (except as noted); item 5 is the ESR derivative peak-to-peak linewidth; items 6 and 7 are the measured (by DNP) and calculated magnetic field separations of the two satellite transitions; items 8 and 9 are the peak positive and negative enhancements measured at the two satellite peaks with maximum microwave power; items 10 and 11 are the mole \%-Sm dilutions in the solution as measured by volume ratios (item 10), and in the crystal as measured by x-ray fluorescence (item 11 ); item 12 is the DNP leakage factor $\left(N_{n} T_{e} / N_{e} T_{n}^{m}\right)$; item 13 is the extrapolated, infinite-power enhancement; item 14 is the DNP half-power parameter; and item (15) is the extrapolated, infinite-power enhancement corrected for DNP leakage (see text). The ideal enhancement $E_{i}$ is 240 in these experiments.

\begin{tabular}{|c|c|c|c|c|c|c|c|c|}
\hline Crystal & 3 & 4 & 4 & 5 & 6 & 6 & 7 & 7 \\
\hline 1. $T(\mathrm{~K})$ & 1.81 & 4.22 & 1.87 & 3.1 & 4.22 & 3.0 & 4.24 & 3.49 \\
\hline 2. $\nu_{e}(\mathrm{GHz})$ & 9.130 & 9.155 & 9.121 & 9.128 & 9.120 & 9.089 & 9.147 & 9.127 \\
\hline 3. $\theta(\mathrm{deg})$ & 7 & 7 & 7 & 6 & 7 & 7 & 7 & 7 \\
\hline 4. $T_{n}^{m}(\mathrm{sec})$ & $225^{2}$ & 15 & 200 & $100^{\mathrm{b}}$ & $15^{b}$ & $108^{\mathrm{b}}$ & 15 & 57 \\
\hline 5. $\Delta H_{\mathrm{pn}}(\mathrm{Oe})$ & $\cdots$ & 5 & 5 & 5 & 5 & 5 & 6 & 6 \\
\hline 6. Measured $\left(H_{+}-H_{-}\right)(\mathrm{Oe})$ & $\cdots$ & 74 & 74 & 74 & 74 & 75 & 74 & 74 \\
\hline 7. Calculated $\left(H_{+}-H_{-}\right)(\mathrm{Oe})$ & $\cdots$ & 74.6 & 74.2 & 74 & 74 & 74 & 74.3 & 74.3 \\
\hline 8. $E_{\max }^{+}$ & 22 & 36 & 38 & 50 & 56 & 147 & 30 & 64 \\
\hline 9. $E_{\max }^{-}$ & $\cdots$ & -40 & -35 & -50 & -56 & -181 & -32 & -79 \\
\hline 10. mole $\% \operatorname{Sm}$ (Sol.) & 15 & 20 & 20 & 25 & 25 & 25 & 20 & 20 \\
\hline 11. mole \% Sm(Xtal. ) & 0.1 & 0.3 & 0.3 & 0.8 & 1.1 & 1.1 & 0.3 & 0.3 \\
\hline 12. $f_{e}^{m}$ & 13.4 & 0.02 & 5.6 & 0.06 & 0.005 & 0.06 & 0.01 & 0.03 \\
\hline 13. $E_{\infty}$ & 24.7 & 199 & $\cdots$ & 81.6 & 189 & 205 & 105 & 121 \\
\hline 14. $P_{1 / 2}$ & 0.095 & 4.46 & $\cdots$ & 0.60 & 2.52 & 0.44 & 2.76 & 0.91 \\
\hline 15. $E_{\infty}\left(1+f_{e}^{m}\right)$ & 355 & 203 & $\geq 250^{c}$ & 86.5 & 190 & 217 & 106 & 125 \\
\hline
\end{tabular}

${ }^{2}$ Estimated from Fig. 1 and from $T_{n}^{m}$ of crystal 4 at $T=1.87 \mathrm{~K}$.

${ }^{b}$ Estimated from Fig. 1.

${ }^{c} E_{\operatorname{mex}}^{+}\left(1+f_{e}^{m}\right)=250 ;$ no $E_{s s}$ vs $P$ data taken.

$f_{d} C_{n} \leqslant 1$. Then Eqs. (21a)-(21c) become

$$
E_{s s}=\left(\frac{\omega_{e}}{\omega_{n}}\right) \frac{\omega_{n} \Delta}{\Delta^{2}+\left(T_{e} / T_{d}\right) \omega_{L}^{2}+f_{e} \omega_{n}^{2}},
$$

which has peak values $E_{s s}^{\text {peak }}$ of

$$
E_{\text {se }}^{\text {peak }}=\left( \pm \frac{\omega_{e}}{\omega_{n}}\right) \frac{\omega_{n}}{2\left[\left(T_{e} / T_{d}\right) \omega_{L}^{2}+f_{e} \omega_{n}^{2}\right]^{1 / 2}}
$$

at $\Delta= \pm\left[\left(T_{\varepsilon} / T_{d}\right) \omega_{L}^{2}+f_{e} \omega_{n}^{2}\right]^{1 / 2}$. Abragam and Borghini, ${ }^{4,7,8}$ Goldman, ${ }^{11}$ and Swanenburg et al. ${ }^{29}$ have discussed this effect for the case of unresolved and partially resolved ${ }^{8}$ satellites, but it appears to be relevant for well-resolved satellites if $S^{d} \gg 1$. The dominating terms are those involving $\left(S^{0} S^{d}\right)$ in Eqs. (21b) and (21c): Saturation of $S^{0}$ cools EDDR, and NZR is then cooled by the $S^{d}$ interaction connecting these two reservoirs. ${ }^{30}$ If $\left(T_{e} / T_{d}\right) \omega_{L}^{2} \gg f_{e} \omega_{n}^{2}$, which may not be easy to satisfy if $\omega_{n} \gg \omega_{L}$, then the peak enhancement values become $^{4} E_{\mathrm{ss}}^{\text {peak }} \approx \pm E_{i}\left(\omega_{n} / 2 \sqrt{2} \omega_{L}\right) \gg E_{i}$ at $\Delta= \pm \sqrt{2} \omega_{L}$, where we take $\left(T_{e} / T_{d}\right)=2$. The key assumption is that the ESR line shape be Lorentzian or Lorentzian-like, so that $S^{d} \gg 1$ holds even when $\omega_{n} \gg \omega_{L}$.
Kittel and Abrahams ${ }^{31}$ have shown that the ESR line shape for dilute paramagnetic systems is approximately Lorentzian for concentrations $<1 \%$, and Gaussian for concentrations $>10 \%$. This suggests that significant homogeneous line broadening with Lorentzian-like wings should occur in the range of $(3-5) \%$ dopings.

The DNP leakage factor $f_{e}^{m}$ can be calculated from measurements of $\left(N_{n} / N_{e}\right), T_{e}$, and $T_{n}^{m}$. If $S^{d} \gg 1$, we found ${ }^{32} T_{n}^{m} \ll T_{n}$, in which case $T_{n}$ and $f_{e}=\left(N_{n} T_{e} / N_{e} T_{n}\right)$ are not directly measurable. It is $f_{e}\left(\ll f_{e}^{m}\right)$ which controls the DNP leakage in Eqs. (23) and (24), as the EDDR contributions to nuclear relaxation do not affect the DNP leakage in that case, contrary to Swanenburg et al. ${ }^{29}$ The physical reason is that the EDDR temperature $\gamma(t)$ is driven far away from $\beta_{L}$ by the ESR microwave photons in DNP, and thus EDDR is not available for regular nuclear relaxation, which is measured under conditions of no ESR, i. e., $S^{0}=0=S^{+}=S^{-}$.

The second possibility for explicit EDDR DNP effects is near, but not at, the satellite peaks with $S^{d} \gg 1, f_{d} C_{n} \leqslant 1, S^{ \pm} \neq 0, S^{0}=0=S^{*}$. Equations (21a)-(21c) then become

$$
E_{s s}=\frac{S^{d}\left(1+f_{d} C_{n}\right)+S^{ \pm}\left[\mp\left(\omega_{\theta} / \omega_{n}\right)\left[1 \mp f_{g} S^{d}\left(\omega_{n} \Delta / \omega_{L}^{2}\right) R\right]\right.}{S^{d}\left(1+f_{d} C_{n}\right)+S^{ \pm}\left[1+f_{e}+f_{d} C_{ \pm}+f_{s} S^{d}\left(C_{\Delta} R+1+f_{d} C_{n}\right)\right]} .
$$




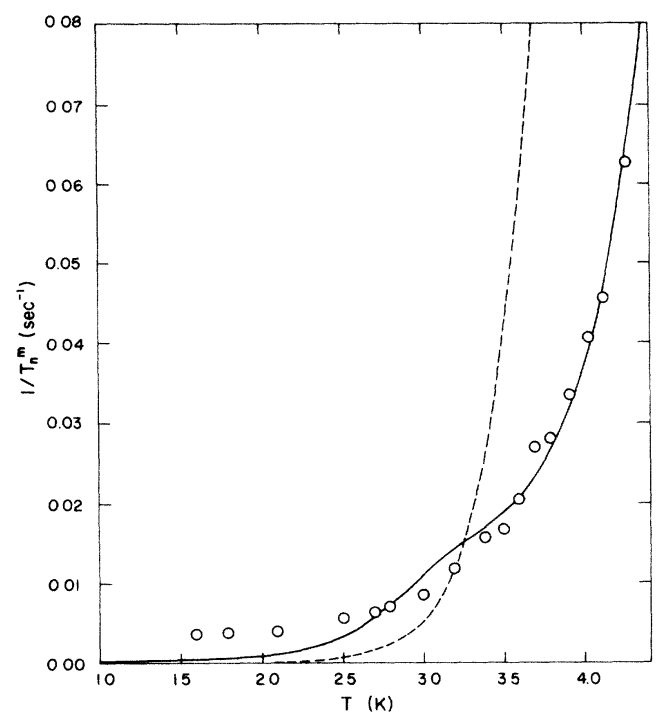

FIG. 1. Measured proton spin-lattice relaxation rate vs temperature for crystal 7 at $H_{0}=8970$ Oe. Circles are data points. Solid line is Eq. (13) in the text using parameters determined by a least-squares fit to the data and tabulated in Table II. Broken line is $\left(1 / T_{n}^{m}\right) \propto\left(1 / T_{e}\right)$, as discussed in the text. $T_{e}$ values were taken from Ref. 17.

If $f_{e} S^{d} \ll 1$, there are negligible EDDR DNP effects, as Eq. (25) reduces to Eq. (16) for all $\Delta$ values near the satellite peaks. However, if $f_{e} S^{d} \geqslant 1, \mathrm{Eq}$. (25) predicts asymmetries in $E_{s s}$ as a function of $\Delta$, as well as higher $E_{\text {ss }}$ values than those in Eq. (16). In this case EDDR is cooled by the satellite transitions, and this is conveyed to NZR by the $W^{d}$ transitions. This proposed new DNP effect, which involves the term $\left(f_{e} S^{ \pm}\right) S^{d}$, is a higher-order analog of the Abragam and Borghini $\left(S^{0} S^{d}\right)$ mechanism ${ }^{30}$ for the main ESR line discussed above. Significant cooling of EDDR by the $S^{ \pm}$ transitions requires that $f_{e}$ be large enough so that $f_{e} S^{d} z 1$, which may be hard to meet in practical experiments. For unresolved satellites the Abragam and Borghini mechanism will predominate, but the two effects are separable for well-resolved satellites.

Finally, we note that the "cross-effect" DNP mechanism of Hwang and $\mathrm{Hill},{ }^{8,33,34}$ does not apply to well-resolved satellites.

\section{EXPERIMENTAL PROCEDURE}

Single crystals of LMN : Sm were grown from mixed, saturated water solutions of SmMN and LMN at $0{ }^{\circ} \mathrm{C}$. As seen in Table I, the Sm dilution (mole \%) measured by $\mathrm{x}$-ray fluorescence is lower by a factor $\approx 50$ than that of the growing solutions, similar to previous results. ${ }^{17,35}$ In the preparation ${ }^{20}$ we used $\mathrm{Sm}_{2} \mathrm{O}_{3}$ and $\mathrm{La}_{2} \mathrm{O}_{3}$ with $99.99 \%$ rare- earth purity; the samarium had the natural isotopic composition. Typical samples weighed 200 mg.

We used conventional nuclear magnetic resonance (NMR), ESR, and cryogenic techniques. ${ }^{1,3,4,20}$ An $X$ band, crystal video, reflection spectrometer was used to measure ESR spectra at low microwave powers, and to excite the DNP with variable power up to a maximum $\approx 0.5 \mathrm{~W}$. The proton $\mathrm{DNP}$, excited at fixed microwave frequency $\nu_{e}$ and variable $H_{0}$, was measured from the ratio of the proton NMR signal peak-to-peak height when polarized to that at thermal equilibrium. $Q$ meter and lock-in detection were used. Because of the poor thermal-equilibrium proton NMR signal, our DNP measurements have an absolute accuracy of $\pm 20 \%$. The relative accuracy of the DNP for $E_{\mathrm{ss}}>10$ is $\pm 5 \%$. Proton spin-lattice relaxation times were measured from the observed NMR decays to thermal equilibrium after the DNP microwaves were turned off. Few measurements of the DNP pump time $\tau_{\mathrm{DNP}}$ were attempted.

\section{EXPERIMENTAL RESULTS}

Single crystals of LMN : Sm have axial symmetry and principal $g$ values ${ }^{35} g_{11}=0.736 \pm 0.005$ and $g_{\perp}=0.363 \pm 0.10$. Scott et al. ${ }^{36}$ have measured the ESR derivative peak-to-peak linewidth $\Delta H_{\mathrm{pp}}$ of a single crystal of 0.05 mole \% LMN : Sm at $1.9 \mathrm{~K}$ and $9.25 \mathrm{GHz}$ as a function of the angle $\theta$ between the magnetic field $\overrightarrow{\mathrm{H}}_{0}$ and the crystal symmetry axis. They found a monotonic increase of the line-

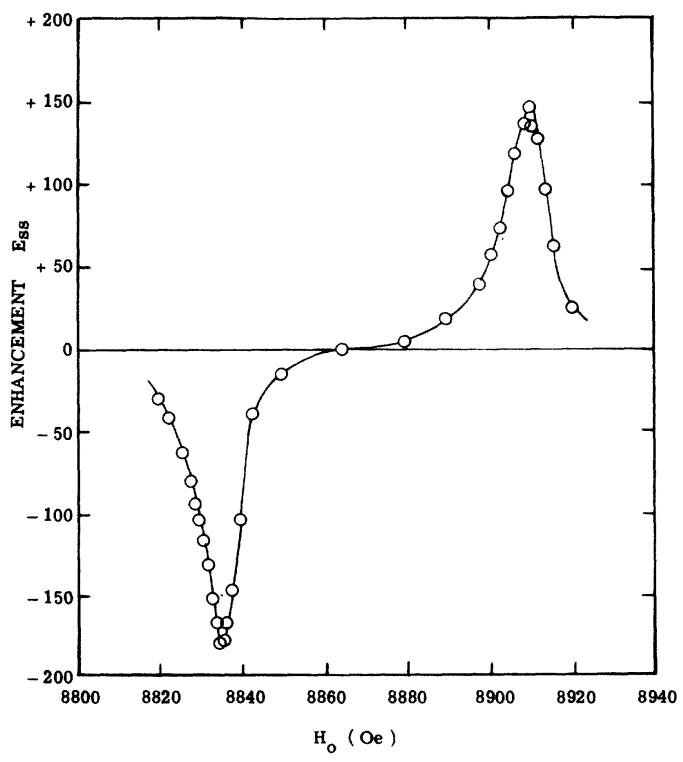

FIG. 2. Enhancement $E_{\mathbf{s s}}$ vs magnetic field for crystal 6 at $3.0 \mathrm{~K}$ with maximum microwave power. The solid line is a smooth curve through the data points. 


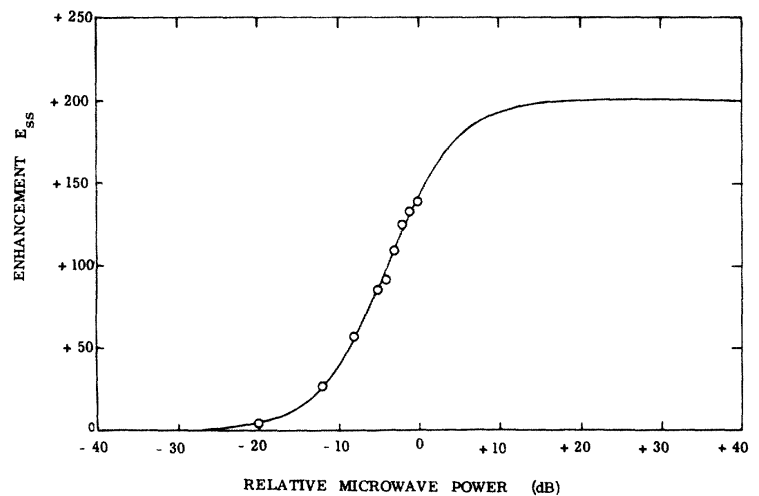

FIG. 3. Enhancement $E_{\text {ss }}$ vs relative microwave power for crystal 6 at $H_{0} \approx 8910 \mathrm{Oe}$ and $T=3.0 \mathrm{~K}$. Circles are data points, and the solid line is Eq. (26) in the text with the values $E_{\infty}=205$ and $P_{1 / 2}=0.44$, determined by a least-squares fit to the data.

width from $\Delta H_{\mathrm{pp}} \approx 4$ Oe at $\theta=0^{\circ}$ to $\Delta H_{\mathrm{pp}} \approx 60$ Oe at $\theta=90^{\circ}$. Thus LMN : Sm has well-resolved satellites near the parallel orientation at $X$ band, since the spacing between the $W^{0}$ peak and the $W^{ \pm}$peaks is $\approx 37 \mathrm{Oe}$ at $\theta=0^{\circ}$ (due to the low $\mathrm{Sm} g$ value). For experimental convenience we operated at $\theta$ $\approx 7^{\circ}$; the measured values of $\Delta H_{\mathrm{pp}}$ for our crystals are given in Table $I$. The electron spin-lattice relaxation time $T_{e}$ of LMN:Sm has been measured by Larson and Jeffries, ${ }^{17}$ who found no evidence for a phonon bottleneck in the parallel orientation.

The main ESR line in LMN:Sm is due to the $71.2 \%$ abundant even-even Sm nuclei. The ions with $14.97 \%$ abundant ${ }^{147} \mathrm{Sm}$ and $13.83 \%$ abundant ${ }^{149} \mathrm{Sm}$ nuclei contribute 16 hyperfine lines which are separated from the main ESR line by hundreds of oersteds. These latter Sm ions do not contribute to the DNP, but are a source of nuclear leakage relaxation, as discussed in Sec. V.

No systematic study of the proton spin-lattice relaxation was attempted. Measurements of $T_{n}^{m}$ are given in Table $I$. In crystal 7 we measured $T_{n}^{m}$ as a function of $T$ at $H_{0}=8970 \mathrm{Oe}$ (Fig. 1). These data will be discussed in Sec. V.

We show the magnetic field dependence of $E_{s s}$ at maximum microwave power in crystal 6 at $3.0 \mathrm{~K}$ in Fig. 2, and the enhancement versus microwave power at the positive enhancement peak $\left(H_{0} \approx 8910\right.$ $\mathrm{Oe})$ in Fig. 3. In no case did we have sufficient microwave power to completely saturate the satellite transitions. Therefore our data have been fitted by the least-squares method to

$$
E_{\mathrm{ss}}=\frac{P_{1 / 2}+P E_{\infty}}{P_{1 / 2}+P}
$$

where $P$ is the variable microwave power in arbi- trary units ( $P=1$ at maximum microwave power $\approx 0.5 \mathrm{~W})$, and $E_{\infty}$ and $P_{1 / 2}$ are constants determined in the fit and tabulated in Table I. The infinite power enhancement is $E_{\infty}$, and $P_{1 / 2}$ is the half-power parameter. We have transformed Eq. (16) into $\mathrm{Eq}$. (26) because the absolute magnitude of $S_{m}^{ \pm}$is not known.

We summarize our data for five crystals in Table I. Other incomplete data for crystals $1,2,5$, and 7 are given in Ref. 20。 The measured maximum enhancements for $P=1$ near the positive and negative enhancement peaks $\left\{\right.$ at $H_{t} \equiv\left(h \nu_{e} / g_{e} \mu_{B}\right)$ $\left.\times\left[1 \pm\left|\omega_{n} / \omega_{e}\right|\right]\right\}$ are labeled $E_{\max }^{ \pm}$. The measured enhancements $E_{\max }^{+}$are extrapolated to infinite microwave power with Eq. (26). We then multiply by $\left(1+f_{e}^{m}\right)$, where we have measured ${ }^{37}\left(N_{n} / N_{e}\right)$ and $T_{n}^{m}$, and $T_{e}$ is taken from Ref。17. If theory and experiment are to agree, $\left[E_{\infty}\left(1+f_{e}^{m}\right)\right]$ must equal the ideal enhancement $\left(\omega_{e} / \omega_{n}\right)$ which is 240 in our experiments.

The magnetic field dependence of $E_{s s}$ near the positive enhancement peak in crystal 4 is shown in Fig. 4. The solid line is the function

$$
E_{\mathrm{ss}}=\frac{P_{1 / 2}+P E_{\infty} f(\Delta H)}{P_{1 / 2}+P f(\Delta H)},
$$

taken from Eqs. (16) and (26), where $f(\Delta H)$ $=\exp \left[-2(\Delta H)^{2} /\left(\Delta H_{\mathrm{pp}}\right)^{2}\right], \Delta H$ is the variable magnetic field referred to the satellite peak, and $E_{\infty}$ and $P_{1 / 2}$ are taken from the crystal 4 data at

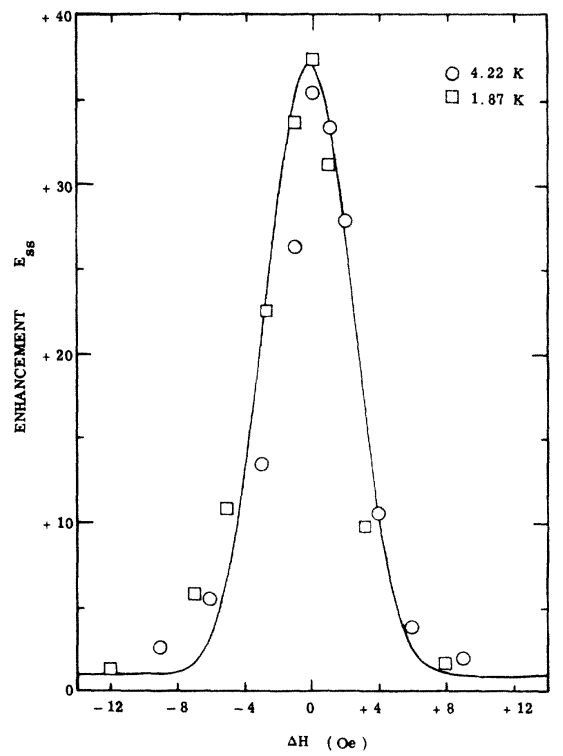

FIG. 4. Enhancement $E_{\text {ss }}$ vs magnetic field near the positive enhancement peak of crystal 4 with maximum microwave power $(P=1)$ at two temperatures. The solid line is Eq. (27) in the text using the values of $\Delta H_{\mathrm{pp}}, E_{\infty}$, and $P_{1 / 2}$ for crystal 4 at $T=4.22 \mathrm{~K}$ in Table I. 
TABLE II. Experimental and theoretical nuclear spinlattice relaxation parameters for crystal 7 .

\begin{tabular}{cll}
\hline \hline & Experiment $^{\mathrm{a}}$ & Theory $^{\mathrm{b}}$ \\
\hline$\frac{3}{10}\left(\frac{g_{e} \mu_{B}}{H_{0}}\right)^{2} \frac{1}{d^{3} R^{3}}$ & $1.1 \times 10^{-6}$ & $3.1 \times 10^{-8}$ \\
$T_{2 e}(\mathrm{sec})$ & $6.5 \times 10^{-5}$ & $1.2 \times 10^{-6}$ \\
$W^{d}\left(\mathrm{sec}^{-1}\right)$ & 0.016 & 0.025 \\
$\frac{N_{n} \omega_{n}^{2}}{N_{e^{\prime} \omega_{L}^{2}}}$ & $1.5 \times 10^{4}$ & $4.7 \times 10^{7}$ \\
\hline
\end{tabular}

${ }^{2}$ Estimated from a least-squares fit of the $T_{n}^{m}$ data in Fig. 1 to Eqs. (8), (10), (11), and (13) in the text, assuming $\left(1 / T_{n}^{L}\right)=0$ and using $T_{e}$ values from Ref. 17 .

${ }^{\mathrm{b}}$ Discussed in the text.

$4.22 \mathrm{~K}$ in Table I. We did not integrate our ESR derivative signal data to obtain an ESR line-shape function, but used a Gaussian instead.

\section{DISCUSSION OF RESULTS}

The nuclear-relaxation data for crystal 7 shown in Fig. 1 were fitted by the least-squares method to Eqs. (8), (10), (11), and (13), assuming ${ }^{38} T_{e}$ $=T_{d}$ and $\left(T_{n}^{L}\right)^{-1}=0$, and using $T_{e}$ values from Ref. 17. The best fit, shown as the solid line in Fig. 1 , generated the experimental parameters given in Table II. The fit was very sensitive to the starting values of the parameters, and does not follow the data points in all respects. We also show a curve proportional to $\left(T_{e}\right)^{-1}$ (broken line in Fig. 1). The values of this curve vary by a factor $\approx 10^{4}$ in the temperature range $1.6-4.2 \mathrm{~K}$, whereas the $T_{n}^{m}$ data points vary by a factor $\approx 16$. This evidently rules out the possibility that the nuclear relaxation is due only to the $\left(T_{n}\right)^{-1}$ term in Eq. (13), i. e., $W^{d}=0$ over the entire temperature range, unless $\left(T_{n}^{L}\right)^{-1}$ effects are not negligible. It also rules out the limit $W^{d} T_{n} \gg\left(N_{n} \omega_{n}^{2} / N_{e} \omega_{L}^{2}\right) W^{d} T_{d} \gg 1$. Unsuccessful attempts were made to fit the data with Eq. (13) for the cases (i) $\left(T_{n}\right)^{-1}=0$, and (ii) $\left(N_{n} \omega_{n}^{2} / N_{e} \omega_{L}^{2}\right) W^{d} T_{d} \ll 1$.

To compare these results with theory, we estimate the theoretical parameters in Table II from our experimental data for ${ }^{39} g_{e}, H_{0}, \omega_{n}, N_{n}$, and $N_{e}$, taking $d=4.36 \AA$. The method of Kittel and Abrahams ${ }^{31}$ was used to calculate $T_{2 e}$ and $\omega_{L}^{2}$ from our measured value of $N_{e}$. We do not get better than an order-of-magnitude agreement between theory and experiment. The values of $\left(N_{n} \omega_{n}^{2} /\right.$ $\left.N_{e} \omega_{L}^{2}\right)$, in particular, disagree significantly. We have assumed that the ${ }^{147} \mathrm{Sm}$ and ${ }^{149} \mathrm{Sm}$ ions do not contribute to the terms containing $W^{d}$, but do contribute to $T_{n}^{Z}$ in the same way as the other Sm ions. Thus the nuclear leakage relaxation $\left(T_{n}^{L}\right)^{-1}$ due to the ${ }^{147} \mathrm{Sm}$ and ${ }^{149} \mathrm{Sm}$ ions is implicitly built into
$T_{n}^{Z}$ in this case. In Sec. IIC we showed theoretically that the DNP leakage factor $f_{e}^{m}$ for LMN : Sm should satisfy the condition $f_{e}^{m} \ll 1$ if $\left(T_{n}^{L}\right)^{-1}$ is negligible. However, our experiments indicate $f_{e}^{m}>1$ in crystals 3 and 4 at $T<2 \mathrm{~K}$ (Table I). This suggests that unknown impurity $\left(T_{n}^{L}\right)^{-1}$ relaxation is important below $T=2 \mathrm{~K}$, although unusual $\left(T_{n}^{L}\right)^{-1}$ effects of the ${ }^{147} \mathrm{Sm}$ and ${ }^{149} \mathrm{Sm}$ ions, or the influence of the inhomogeneous broadening contributions (see below) to the LMN: Sm ESR line, may be involved, too. On the other hand, if one views the fit of our $T_{n}^{m}$ data to Eq. (13) in Fig. 1 as being reasonably good, and thus overlooks the discrepancies in Table II, this suggests that EDDR $W^{d}$ processes may have had some influence on the nuclear-relaxation rate $\left(1 / T_{n}^{m}\right)$ below $T=3 \mathrm{~K}$, in which case $S^{d} \gg 1$ at $T<2 \mathrm{~K}$. However, no definite conclusion can be reached on the basis of our experiments.

We summarize the results given in Table I by noting that the peak enhancements of crystals 3,4 , and 6 are in general agreement with Eqs. (16) and (26), and that those of crystals 5 and 7 are not. This is most easily seen by comparing $\left[E_{\infty}\left(1+f_{e}^{m}\right)\right]$, item 15 in Table I, with the ideal enhancement $E_{i}$ $=240$ for these experiments. Agreement is within the $\pm 20 \%$ absolute accuracy for three of the crystals. ${ }^{40}$ It is significant that a given crystal either agrees or disagrees at all temperatures measured. Items 12 and 14 in Table I demonstrate the principal difficulties in interpreting our experiments. At higher temperatures $(T \approx 4.2 \mathrm{~K})$ where $f_{e}^{m} \ll 1$, we had insufficient microwave power $\left(P_{1 / 2}>2.5\right)$ to obtain $E_{\max }^{ \pm} \approx E_{\infty} \approx E_{i}$, while at lower temperatures $(T \approx 1.8 \mathrm{~K})$ where the maximum power was adequate $\left(P_{1 / 2} \ll 1\right)$, the large DNP leakage factor $f_{e}^{m}$ $(\approx 5-13)$ greatly reduced $E_{\infty} . T_{n}^{m}$ was not measured in crystals 3,5 , and 6 , which is a source of concern in the $f_{e}^{m}$ estimation. The $T_{e}$ values used in calculating $f_{e}^{m}$ in all cases were taken from Ref. 17, and were not measured for our crystals, which introduces errors that are difficult to estimate, especially in the $T \approx 1.8 \mathrm{~K}$ data.

We have no conclusive explanation for the discrepancy between $E_{i}$ and $E_{\infty}\left(1+f_{e}^{m}\right)$ in crystals 5 and 7. Since $f_{e}^{m} \leqslant 0.06$ in all our data for $T>3 \mathrm{~K}$, errors in estimating $f_{e}^{m}$ seem an unlikely explanation. If the Sm ions formed clusters in the LMN host lattice instead of occupying sites at random, then one might expect anomalous DNP results. However, we have no measurements showing the existence or nonexistence of Sm clusters. The absence of a phonon bottleneck in the work of Larson and Jeffries ${ }^{17}$ on LMN : Sm suggests that this is not an explanation for reduced DNP enhancements. ${ }^{2,3,5,6}$ The possibility that inhomogeneous broadening of the ESR line reduces the DNP is discussed below. 
The measured values of $E_{\max }^{+}$and $E_{\max }^{-}$were the same within experimental error for each crystal at each temperature reported here except for crystal 6 at $T=3.0 \mathrm{~K}$ and crystal 7 at $T=3.49 \mathrm{~K}$. Inequality of the two peaks has been observed before and was attributed to drifts in the instrumentation. ${ }^{1}$ We assume that to be the case here. The calculated and measured separations of the satellite peaks (items 6 and 7 in Table I) are in good agreement.

Both the $4.22 \mathrm{~K}$ and $1.87 \mathrm{~K}$ enhancement data of crystal 4 fitted Eq. (27) with a Gaussian function using the measured $\Delta H_{\mathrm{pp}}$, as seen in Fig. 4. The solid line drawn for the $T=4.22-\mathrm{K}$ values of $E_{\infty}$ and $P_{1 / 2}$ is very sensitive to the value of $\Delta H_{p p}$, and the agreement with the $E_{\text {ss }}$ data disappears if $\Delta H_{\mathrm{pp}}$ is given a small variation $\approx 1$ Oe.

There is no evidence in Figs. 2 and 4 for the EDDR DNP effects predicted in Eqs. (23)-(25). The enhancement is unity near the main ESR transition $W^{0}$, and there are no asymmetries in the DNP near $H_{ \pm}$, even at $T<2 \mathrm{~K}$, where $S^{d}$ may be $\gg 1$. This was true for all our crystals at 9.1 $\mathrm{GHz}$, and in addition is consistent with $70 \mathrm{GHz}$, well-resolved satellite DNP results of Schmugge and Jeffries ${ }^{1}$ in LMN : Nd and of Wollan and Stapleton $^{10}$ in YES : Nd (neodymium-doped yttrium ethyl sulphate). There are several possible reasons to explain this. First, the appropriate limits $\left(S^{d}\right.$ $\gg 1, f_{e} S^{d} \gg 1$ ) for the two cases may not have been obtained even at $T \approx 1-2 \mathrm{~K}$, e.g., if the ESR line shape was not sufficiently Lorentzian-like in the wings, or if $\left(T_{e} / T_{2 e}\right) \ll 1$ [using Eq. (12)]. Second, if the ESR lines are not completely homogeneously broadened, the assumptions of single-spin temperatures $\alpha(t)$ and $\gamma(t)$ for the entire electron system and of a single EDDR with a large heat capacity, are not valid. It is thus necessary to estimate either experimentally or theoretically the homogeneous and inhomogeneous broadening ${ }^{23}$ contributions to the ESR line shape. In crystal 4, our DNP data are fitted well by a Gaussian with $\Delta H_{p p}$ $=5$ Oe (Fig。4). We have used the Kittel and Abrahams ${ }^{31}$ method to estimate theoretically that the homogeneous broadening due to the $\mathrm{Sm}$ ions in a 0.3-mole \% LMN : Sm crystal corresponds to a cut-off Lorentzian line shape with a full width at half-maximum $\approx 0.35$ Oe. This suggests that the ESR line shape was largely inhomogeneously broadened, in which case the EDDR aspects of the theory in Sec. II would not apply. Wollan and Poul$\operatorname{ton}^{41}$ have shown that the effective leakage factor is greater than $f_{e}^{m}$ for DNP with an inhomogeneously broadened ESR line. This might explain the discrepancy between the DNP data and Eqs. (16) and (26) for crystals 5 and 7. Because of the high rejection ratio of the Sm ions in LMN : Sm aqueous solutions, efforts to grow crystals $\approx 3-5-$ mole $\%$ LMN : Sm have so far been unsuccessful. We believe that the EDDR DNP effects should be observable in such crystals at $T<2 \mathrm{~K}$.

\section{SUMMARY}

We have derived theoretical expressions for the dynamic nuclear polarization and nuclear spinlattice relaxation for ionic crystals weakly doped with paramagnetic impurities assuming (i) a completely homogeneously broadened ESR line, (ii) an isotropic electron $g$ factor, (iii) rapid nuclear spin diffusion, (iv) the high-temperature limit, and (v) no phonon bottleneck. In the limit of well-resolved satellites, the conventional results for DNP at the satellite peaks are obtained, but with EDDR effects on the nuclear relaxation rate and DNP leakage factor included. We suggest that direct EDDR contributions to DNP excited by saturation of the main ESR line ${ }^{4}$ may be extended to crystals with well-resolved satellites in some cases. We propose a new DNP effect: Saturation of the satellite transitions may cool EDDR in favorable cases, and lead to asymmetries in the shape of the DNP curve as well as slightly higher polarizations than those at the satellite peaks.

Our experimental DNP results for three 0.1-1.1mole \% LMN : Sm crystals agree with the conventional theory at the satellite peaks. Data for two other crystals disagree with theory; some reasons are proposed, none conclusive. No experimental evidence for direct EDDR effects on the DNP either near the main ESR line or near the satellites was found, despite nuclear relaxation data suggesting that the conditions may have been favorable at $T<2 \mathrm{~K}$. Possible explanations are proposed. We suggest that these EDDR effects may be seen in crystals of $\approx 3-5-$ mole \% LMN : Sm, which we have so far been unable to grow, or in equivalent systems. In our experiments EDDR may have had some influence on the nuclear spinlattice relaxation, but had no direct effect on the dynamic nuclear polarization.

\section{ACKNOWLEDGMENTS}

We thank H. C. Ballagh, Jr. and Professor D. Kaplan for help on aspects of the theory, and Professor R. A. Arndt for aid in the computer calculations.
*This paper is based in part upon a dissertation submitted by Charles E. Byvik in partial fulfillment of the requirements for the $\mathrm{Ph}$. D. degree at the Virginia Polytechnic Institute and State University.
${ }^{1}$ T. J. Schmugge and C. D. Jeffries, Phys. Rev. 138, A1785 (1965).

${ }^{2}$ C. D. Jeffries, Technical Report No. UCB-34P20-T-1, Physics Department, University of California, 1966 
(unpublished).

${ }^{3}$ C. D. Jeffries, in Electron Paramagnetic Resonance, edited by S. Geschwind (Plenum, New York, 1972), pp. 217-262.

${ }^{4} \mathrm{~A}$. Abragam and M. Borghini, in Progress in Low Temperature Physics, edited by C. J. Gorter (North-Holland, Amsterdam, 1964), Vol. 4, pp. 384-449.

${ }^{5}$ M. Borghini, Phys. Rev. Lett. 16, 318 (1966).

${ }^{6} \mathrm{M}$. Borghini, Phys. Lett. 20, 228 (1966).

${ }^{7}$ M. Borghini, Phys. Lett. 26A, 242 (1968).

${ }^{8} \mathrm{M}$. Borghini, in Proceedings of the Second International Conference on Polarized Targets, Lawrence Berkeley Laboratory Report No. LBL-500, UC-34, TID-4500 1971 (unpublished), pp. 1-32.

${ }^{9}$ G. R. Khutsishvili, Usp. Fiz. Nauk. 87, 211 (1965) [Sov. Phys. - Usp. 8, 743 (1966)].

${ }^{10}$ D. S. Wollan and H. J. Stapleton, Phys. Rev. $\underline{163}, 207$ (1967).

${ }^{11} \mathrm{M}$. Goldman, Spin Temperature and Nuclear Magnetic Resonance in Solids (Oxford U. P., Oxford, 1970).

${ }^{12}$ W. E. Blumberg, Phys. Rev. 119, 79 (1960).

${ }^{13}$ G. R. Khutsishvili, Usp. Fiz. Nauk. 96, 441 (1968) [Sov. Phys. -Usp. 11, 802 (1969)].

${ }^{14} \mathrm{G}$. R. Khutsishvili, in Progress in Low Temperature Physics, edited by C. J. Gorter (North-Holland, Amsterdam, 1970), Vol. 6, pp. 375-404.

${ }^{15}$ G. M. Van den Heuvel, T. J. B. Swanenburg, and N. J. Poulis, Physica 56, 365 (1971).

${ }^{16}$ I. J. Lowe and D. Tse, Phys. Rev. 166, 279 (1968).

${ }^{17}$ G. H. Larson and C. D. Jeffries, Phys. Rev. 141, 461 (1966); 145, 311 (1966).

${ }^{18}$ A. G. Redfield, Phys. Rev. 98, 1787 (1955).

${ }^{19}$ B. N. Provotorov, Zh. Eksp. Teor. Fiz. 41, 1582 (1961) [Sov. Phys. -JETP 14, 1126 (1962)].

${ }^{20} \mathrm{C}$. E. Byvik, Ph. D. dissertation (Virginia Polytechnic Institute and State University, Blacksburg, 1971) (unpublished).

${ }^{21}$ D. S. Wollan, Rev. Sci. Instrum. 42, 682 (1971).

${ }^{22} \mathrm{~A}$. Abragam, The Principles of Nuclear Magnetism (Oxford U. P., Oxford, 1961).

${ }^{23}$ A. M. Portis, Phys. Rev. 91, 1071 (1953).

${ }^{24} \mathrm{Eq}$. (6) is correct to within a factor $\approx 3$. The function $g(\omega)$, which is given in Refs. 11,20 , and 22, depends on terms like $S_{s}^{i} S_{\varepsilon}^{j}$ and $S_{ \pm}^{i} S_{\mp}^{j}$ in $\mathcal{J C}_{S S}^{0}$, where the spin operators $S_{z}^{i}$ and $S_{ \pm}^{i}=S_{x}^{i} \pm i S_{y}^{i}$ refer to the $i$ th electron. $W^{d}$ depends only on the $S_{t}^{i} S_{\mp}^{j}$ terms in $\mathfrak{H C}_{S S}^{0}$. See Ref. 20 and Ref. 22, Chap. 4.

${ }^{25}$ E. P. Horvitz, Phys. Rev. B 3, 2868 (1971).

${ }^{26}$ A. R. King, J. P. Wolfe, and $\vec{R}$. L. Ballard, Phys. Rev. Lett. 28, 1099 (1972).

${ }^{27}$ We should use $\tau \approx(3) T_{2 e}$ instead of $T_{2 e}$ in Eq. (11) for the reason given in Ref. 24. However, Eq. (11) is adequate for the approximate treatment made here.

${ }^{28} E_{i}=\left(\mp \omega_{e} / \omega_{n}\right)$ holds only at high temperatures. In general, $E_{i}=\mp\left[\tanh \left(\hbar \omega_{e} / 2 k T\right)\right] /\left[\tanh \left(\hbar \omega_{n} / 2 k T\right)\right]$, as shown in Refs. 1-3 and 5 .

${ }^{29}$ T. J. B. Swanenburg, G. M. Van den Heuvel, and N. J. Poulis, Physica 33, 707 (1967); 35, 369 (1967); 37, 65 (1967).

${ }^{30} \mathrm{For}$ the case of unresolved satellites, the driving term is $S^{0}\left(S^{d}+S^{+}+S^{-}\right)$. See Eqs. (21a)-(21c) and Refs. 4, 7, 8 , and 11 .

${ }^{31}$ C. Kittel and E. Abrahams, Phys. Rev. 90, 238 (1953).

${ }^{32}$ This is not true for the special limit of $S_{d} \gg 1, f_{d} C_{n} \gtrsim 1$.

${ }^{33}$ C. F. Hwang and D. A. Hill, Phys. Rev. Lett. $\underline{18}, 110$ (1967); 19, 1011 (1967).

${ }^{34} \mathrm{D}$. A. Hill, Ph. D. dissertation (Northwestern University, Evanston, 1970) (unpublished).

${ }^{35}$ P. L. Scott and C. D. Jeffries, Phys. Rev. 127, 32 (1962).

${ }^{36}$ P. L. Scott, H. J. Stapleton, and C. Wainstein, Phys. Rev. 137, A71 (1965). Only the inhomogeneous broadening contributions to $\Delta H_{p p}$ were considered in this paper.

${ }^{37}$ In the estimation of $f_{e}^{m}$, only the $71.2 \% \mathrm{Sm}$ ions in the main ESR line are counted in $N_{e}$.

${ }^{38}$ One often takes $T_{e} \approx(2-3) T_{d}$, as in Ref. 4. Our fit is not sufficiently good to preserve this distinction.

${ }^{39}$ At angle $\theta, g_{e}=\left(g_{\| 1}^{2} \cos ^{2} \theta+g_{\perp}^{2} \sin ^{2} \theta\right)^{1 / 2}$.

${ }^{40}$ Item 15 in Table I for crystal 3 is $50 \%$ higher than $E_{i}$, probably because $T_{n}^{m}$ and $T_{e}$ were taken from data on other crystals.

${ }^{41}$ D. S. Wollan and J. W. Poulton, Phys. Lett. $\underline{33 \mathrm{~A}}, 33$ (1970). 